\title{
First-Principles Study of the Structural, Electronic, Magnetic and Thermal Properties of the Cr Doped $\mathrm{Ge}_{6} \mathrm{Mn}_{2} \mathrm{Te}_{8}$ and $\mathrm{Ge}_{6} \mathrm{Fe}_{2} \mathrm{Te}_{8}$ Systems
}

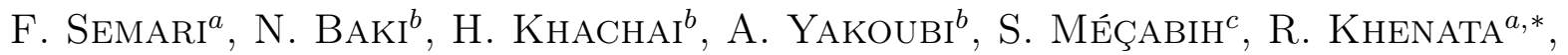 \\ A. ShankAR ${ }^{d}$, D.P. RAI ${ }^{e}$ AND A. BOUHEMADOU ${ }^{f}$ \\ ${ }^{a}$ Laboratoire de Physique Quantique de la Matiére et de Modélisation Mathématique (LPQ3M), \\ Université de Mascara, 29000 Mascara, Algeria \\ ${ }^{b}$ Laboratoire d'Étude des Matériaux et Instrumentations Optiques-Faculté des Sciences Exactes, \\ Djillali Liabès University, 22000 Sidi Bel Abbès, Algeria \\ ${ }^{c}$ Modelling and Simulation in Materials Science Laboratory, Physics Department, University of Sidi Bel-Abbes, \\ 22000 Sidi Bel-Abbes, Algeria \\ ${ }^{d}$ Department of Physics, University of North Bengal, Darjeeling-734013, India \\ ${ }^{e}$ Department of Physics, Pachhunga University College, Aizawl-796001, India \\ ${ }^{f}$ Laboratory for Developing New Materials and Their Characterization, Department of Physics, Faculty of Science, \\ University of Setif 1, Setif 19000, Algeria
}

(Received June 6, 2016; in final form May 22, 2017)

First-principles calculations have been used to study the structural, electronic, magnetic, and thermal properties of the Cr doped $\mathrm{Ge}_{6} \mathrm{Mn}_{2} \mathrm{Te}_{8}$ and $\mathrm{Ge}_{6} \mathrm{Fe}_{2} \mathrm{Te}_{8}$ systems. The calculations were performed using the full-potential linearized augmented plane wave plus local orbitals (FP-LAPW + LO) method based on the spin-polarized density functional theory. Additionally, the electronic exchange-correlation potential is approximated using the spin generalized gradient approximation. The structural properties of the $\mathrm{Ge}_{5} \mathrm{Mn}_{2} \mathrm{CrTe}_{8}$ and $\mathrm{Ge}_{5} \mathrm{Fe}_{2} \mathrm{CrTe}_{8}$ alloys are indicated by their corresponding lattice constants, values of the bulk moduli and their pressure derivatives. An analysis of the band structures and the densities of states indicate that for both alloys, they present nearly halfmetallic ferromagnetism character. The band structure calculations are used to estimate the spin-polarized splitting energies, $\Delta_{x}(d)$ and $\Delta_{x}(p d)$ produced by the $3 d \mathrm{Mn}, 3 d \mathrm{Fe}$ and $3 d \mathrm{Cr}$ doped states as well as the $s(p)-d$ exchange constants, $N_{0} \alpha$ (conduction band) and $N_{0} \beta$ (valence band). It is observed that the $p-d$ hybridization reduces the magnetic moment of the $\mathrm{Mn}$ and Fe atoms from their atomic charge values and create small local magnetic moments on the nonmagnetic Ge and Te sites. Furthermore, the calculations of the charge density indicate that both compounds have ionic bonding character. Through the quasi-harmonic Debye model, the effects of pressure $P$ and temperature $T$ on the bulk modulus $B$, the primitive cell volume $V / V_{0}$, the Debye temperature $\theta_{\mathrm{D}}$, the Grüneisen parameter $\gamma$, the heat capacity $C_{V}$, the entropy $S$, as well as the thermal expansion coefficient, $\alpha$ of the $\mathrm{Ge}_{6} \mathrm{Mn}_{2} \mathrm{Te}_{8}, \mathrm{Ge}_{5} \mathrm{Mn}_{2} \mathrm{CrTe}_{8}, \mathrm{Ge}_{6} \mathrm{Fe}_{2} \mathrm{Te}_{8}$ and $\mathrm{Ge}_{5} \mathrm{Fe}_{2} \mathrm{CrTe}_{8}$ alloys are predicted.

DOI: 10.12693/APhysPolA.132.1242

PACS/topics: first-principles calculations, half-metallic ferromagnetism, electronic properties, magnetic moment, spin-exchange splitting, thermodynamic properties

\section{Introduction}

The diluted magnetic semiconductors (DMS) are one of the most promising and interesting classes of magnetic materials. Due to the exchange interaction between the carriers and the magnetic impurities, the bulk DMS have interesting magnetic and magneto-optical properties $[1,2]$. The signature characteristic of the DMS is the carrier-mediated ferromagnetic exchange where the magnetic and electronic properties of the microelectronic devices can be linked [3]. Transition metal doped II-VI and III-V diluted magnetic semiconductors have many unique magneto-optical, magneto-electrical and

*corresponding author; e-mail: khenata_rabah@yahoo.fr magneto-transport properties that are essential for the future generation of the spintronic device applications [4].

Recently, GeTe thin films doped with the $3 d$ transition metals have received considerable attention from experimentalists interested in the field of magnetic semiconductors $[5,6]$. Ferromagnetic order was observed for the $\mathrm{Cr}, \mathrm{Mn}$, and $\mathrm{Fe}$ doped films, where the $\mathrm{Ti}, \mathrm{V}, \mathrm{Co}$, and $\mathrm{Ni}$ doped films were found to be paramagnetic [7]. The Curie temperatures $T_{\mathrm{C}}$ of these thin films have been found to depend on the type and concentration of the dopants with a high value of $140 \mathrm{~K}$, reported for the $\mathrm{Ge}_{1-x} \mathrm{Mn}_{x}$ Te alloys at $x=0.51$ [6]. Later on this group of researchers reported even higher value of $\mathrm{T}_{\mathrm{C}} \approx 190 \mathrm{~K}$ in the DMS $\mathrm{Ge}_{0.92} \mathrm{Mn}_{0.08} \mathrm{Te}[8]$ alloy where $T_{\mathrm{C}}$ 's around $200 \mathrm{~K}$ to $250 \mathrm{~K}$ have been reported by Fukuma et al. [9]. For the $\mathrm{Ge}_{1-x} \mathrm{Cr}_{x}$ Te thin films at low values of $x(\leq 0.1)$ the thin films in all these studies were predominantly of 
the rock salt structure. In addition, for spintronic applications, the desired properties of such ferromagnets are half-metallicity with relatively high Curie temperatures. The possibility of half-metallicity in the Cr- and Vsubstituted GeTe bulk compounds has been reported by Zhao et al. [10] based on the theoretical calculations using the linearized augmented plane wave (LAPW) method in the WIEN2k code for some ordered $\mathrm{Ge}-\mathrm{V}-\mathrm{Te}$ and $\mathrm{Ge}$ $\mathrm{Cr}-\mathrm{Te}$ compounds. However, these authors had left the issue of the Curie temperature unaddressed, as only the electronic band structure, density of states and charge density were studied where the exchange interaction in these compounds were not investigated. A theoretical study of the electronic structure of the $\mathrm{Ge}_{1-x} \mathrm{Mn}_{x}$ Te alloys, based on the generalized gradient approximation plus Hubbard U (GGA + U) has been presented by Ciucivara et al. [11] where among the magnetic properties only the magnetic moment was considered and the issue of the exchange interaction and $T_{\mathrm{C}}$ was not addressed.

Recently a large number of experimental studies related to the electronic structure [12-14], magnetism and magnetic transitions in several Ge-Te based alloys, such as $\mathrm{Ge}-\mathrm{Cr}-\mathrm{Te}, \mathrm{Ge}-\mathrm{Mn}-\mathrm{Te}$ and $\mathrm{Ge}-\mathrm{Fe}-\mathrm{Te}$ have been reported [15-17]. In addition, there is a substantial amount of work on the DMS in general (please refer to Ref. [18] for a review), where the magnetic properties arise via the percolation effects among a very small concentration of the magnetic atoms. For a discussion on the magnetic percolation in DMS, the readers may consult the work by Bergqvist et al. [19]. In view of the large body of existing experimental work as mentioned above, Liu et al. [20] have embarked on a systematic theoretical study of the GeTe system doped with the $3 d$ transition metal atoms where the most commonly occurring structure for both the bulk and thin films of these compounds is the $\mathrm{NaCl}$ or the rock-salt (RS) structure.

On the other hand, a number of research groups performed a series of investigations involving the electronic structure and magnetic properties of ternary transitionmetal compounds based on the rock-salt IV-VI GeTe semiconductor material systems such as the $\mathrm{Ge}_{3} \mathrm{XTe}_{4}$ and $\mathrm{GeXTe}_{2}(\mathrm{X}=\mathrm{Ti}, \mathrm{V}, \mathrm{Cr}, \mathrm{Mn}, \mathrm{Fe}, \mathrm{Co}$ and $\mathrm{Ni})$ alloys. They studied the material systems theoretically using the $a b$ initio calculations in order to explore new half-metallic ferromagnets based on the RS structure [10, 17]. In recent times the calculated exchange interactions due to the carriers originating from these causes are sufficient to account for the observed ferromagnetism in the thin films of $\mathrm{Ge}_{1-x} \mathrm{Mn}_{x} \mathrm{Te}$, such as due to the Mn-Mn exchange interactions in the $\mathrm{RS} \mathrm{Ge}_{0.75} \mathrm{Mn}_{0.25} \mathrm{Te}_{x} \mathrm{Z}_{1-x}$ alloys where $\mathrm{Z}$ represents the Sn-atoms or vacancies. Furthermore, the Sn-atoms or vacancies occupy random positions solely in the Te-sublattice [20].

The objective of the present work is to investigate the structural, elastic, electronic structure, magnetic, and thermal properties of the $\mathrm{Cr}$ doped $\mathrm{Ge}_{6} \mathrm{Mn}_{2} \mathrm{Te}_{8}$ and $\mathrm{Ge}_{6} \mathrm{Fe}_{2} \mathrm{Te}_{8}$ systems using the first-principles full potential linearized augmented plane wave plus local orbitals (FP-
LAPW + LO) method within the spin generalized gradient approximation (spin-GGA). We observed from the calculations that both alloys exhibit nearly half-metallic ferromagnetism character. The remainder of the paper is organized as follows: Sect. 2 describes the calculation method, Sect. 3 presents the results and a discussion of the structural, electronic, magnetic, bonding and thermal properties of the alloys and finally, Sect. 4 summarizes the conclusions of this work.

\section{Computational details}

In this work, we have employed the density functional theory (DFT) to investigate the atomic, electronic and magnetic properties of the $\mathrm{Cr}$ doped $\mathrm{Ge}_{6} \mathrm{Mn}_{2} \mathrm{Te}_{8}$ and $\mathrm{Ge}_{6} \mathrm{Fe}_{2} \mathrm{Te}_{8}$ systems. For the calculations of these properties, we employed the FP-LAPW + LO method as implemented in the Wien2k code [21], which is considered as an efficient method for the accurate calculations and simulations of the ground state properties of materials $[22,23]$. The spin-GGA parametrized by Perdew, Burke and Ernzerhof (PBE) [24] was employed to handle the exchange and correlation effects. In order to obtain the equilibrium structural parameters, we carried out optimization of the total energy with respect to the different values of the unit cell volume using the Murnaghan equation of state [25]. The calculations were performed with $R_{M T}^{*} K_{\max }=8$, which determines the matrix size in order to achieve energy eigenvalue convergence, where $R_{M T}$ is the smallest radius of the muffin-tin (MT) spheres and $K_{\max }$ is the maximum value of the wave vector. The values of the muffin-tin radii $\left(R_{M T}\right)$ for the $\mathrm{Ge},(\mathrm{X}=\mathrm{Mn}$, $\mathrm{Fe}), \mathrm{Cr}$ and $\mathrm{Te}$ atoms were taken to be 2.0, 2.1, 2.1, 2.1 and 2.1 a.u. (atomic units), respectively, for all the calculations. Additionally, the maximum value of the angular momentum, $l_{\max }$ is set to 10 for the wave function expansion inside the atomic spheres and the $k$-points used in the calculations were based on the $7 \times 7 \times 7$ MonkhorstPack scheme [26]. The iteration process was repeated until the calculated total energy and charge of the calculations converged to less than $0.0001 \mathrm{Ry}$. In order to simulate the ordered RS structure (space group, F43m), we have built a GeTe supercell with 16 atoms, and substituted two Ge atoms with one ( $\mathrm{Mn}$ and $\mathrm{Fe}$ ) atom. respectively, and by doping the alloys with one $\mathrm{Cr}$ atom this corresponds to a dopant and carrier concentration of $25 \%$ in the alloys. The Ge and Te atoms are located at the lattice coordinates of $(0,0,0)$ and $(0.50,0.50$, 0.50 ), respectively (see Ref. [18]). Conversely, the investigations of the thermal effects were performed using the quasi-harmonic Debye model as implemented in the Gibbs program [27] to determine all the thermodynamic parameters as a function of temperature and pressure.

\section{Results and discussions}

\subsection{Structural properties}

In order to simulate the $\mathrm{Ge}_{5} \mathrm{Mn}_{2} \mathrm{CrTe}_{8}$ and $\mathrm{Ge}_{5} \mathrm{Fe}_{2} \mathrm{CrTe}_{8}$ quaternary alloys, we have used a $(1 \times 1 \times 1)$ GeTe supercell with 16 atoms and the replacement of the $\mathrm{Ge}$ atoms by the ( $\mathrm{Mn}$ and $\mathrm{Fe}$ ) atoms 
in GeTe to create the $\mathrm{Ge}_{6} \mathrm{Mn}_{2} \mathrm{Te}_{8}$ and $\mathrm{Ge}_{6} \mathrm{Fe}_{2} \mathrm{Te}_{8}$ alloys, and by doping the alloys with one $\mathrm{Cr}$ atom which corresponds to a dopant and carrier concentration of $25 \%$. In order to calculate the ground states properties for both quaternary alloys, we optimize the total energy as a function of the unit cell volume where we have computed the lattice constants, bulk moduli and the first pressure derivatives of the bulk moduli by fitting the total energy versus volume according to the Murnaghan equation of state [25]. Furthermore, we have used the GGA schemes for better visualization of the efficiency and improvement of this approximation on the various properties of the alloys, which is to verify the influence of these corrections on the structural and electronic properties. We summarized our results and the experimental values of the structural properties of the alloys in Table I.

TABLE I

The calculated lattice constant $a_{0}$, bulk modulus $B_{0}$ and its pressure derivative $B_{0}^{\prime}$ of the $\mathrm{Ge}_{5} \mathrm{Mn}_{2} \mathrm{CrTe}_{8}$ and $\mathrm{Ge}_{5} \mathrm{Fe}_{2} \mathrm{CrTe}_{8}$ ferromagnetic alloys.

\begin{tabular}{c|c|c|c|c|c}
\hline \hline \multirow{2}{*}{ Compound } & \multicolumn{2}{|c|}{$a_{0}[\AA]$} & \multicolumn{2}{|c|}{$B_{0}[\mathrm{GPa}]$} & $B_{0}^{\prime}$ \\
\cline { 2 - 6 } & this work & others & this work & others & this work \\
\hline $\mathrm{Ge}_{6} \mathrm{Mn}_{2} \mathrm{Te}_{8}$ & 5.95 & $5.96^{[18]}$ & 49.72 & $49.7^{[18]}$ & 3.51 \\
$\mathrm{Ge}_{5} \mathrm{Mn}_{2} \mathrm{CrTe}_{8}$ & 5.88 & - & 49.86 & - & 3.54 \\
$\mathrm{Ge}_{6} \mathrm{Fe}_{2} \mathrm{Te}_{8}$ & 5.90 & $5.87^{[10]}$ & 46.88 & - & 3.81 \\
$\mathrm{Ge}_{5} \mathrm{Fe}_{2} \mathrm{CrTe}_{8}$ & 5.81 & - & 50.31 & - & 3.90
\end{tabular}

\subsection{Electronic properties}

\subsubsection{Spin-polarized band structure energies}

The obtained equilibrium lattice parameters are used in order to calculate the band structures of the ferromagnetic $\mathrm{Ge}_{5} \mathrm{TM}_{2} \mathrm{CrTe}_{8}$ and $\mathrm{Ge}_{5} \mathrm{Fe}_{2} \mathrm{CrTe}_{8}$ compounds where the spin directions $(\uparrow$ and $\downarrow$ ) are taken as the directions of the transition metal (TM) spins (majority $\uparrow$ and minority $\downarrow$ spin directions). The full spin-polarized electronic band structures of the $\mathrm{Ge}_{5} \mathrm{Mn}_{2} \mathrm{CrTe}_{8}$ alloy within the GGA (in the majority $\uparrow$ and minority $\downarrow$ spin directions) are shown in Fig. 1a and $\mathrm{b}$ as well as the $\mathrm{Ge}_{5} \mathrm{Fe}_{2} \mathrm{CrTe}_{8}$ alloy (in the majority $\uparrow$ and minority $\downarrow$ spin directions) within the GGA are shown in Fig. 2a and b, respectively. It is observed in Figs. 1a and $\mathrm{b}$ that the majority bands cross the Fermi level in almost all the directions of high symmetry, whereas the minority bands exhibit an indirect band gap of $E_{g}=0.43 \mathrm{eV}$, hence confirming the
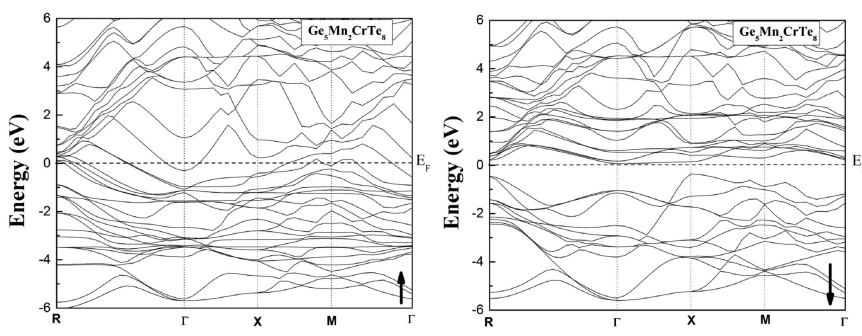

Fig. 1. The spin-polarized band structures of the $\mathrm{Ge}_{5} \mathrm{Mn}_{2} \mathrm{CrTe}_{8}$ alloy (majority $\uparrow$ and minority $\downarrow$ spin directions).
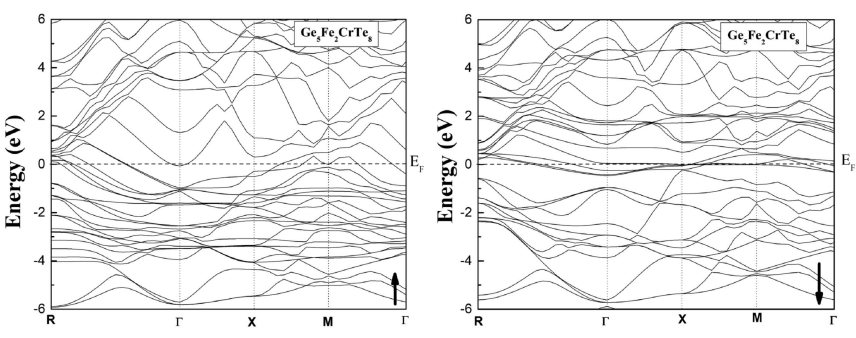

Fig. 2. The spin-polarized band structures of the $\mathrm{Ge}_{5} \mathrm{Fe}_{2} \mathrm{CrTe}_{8}$ alloy (majority $\uparrow$ and minority $\downarrow$ spin directions).

half-metallic character of the $\mathrm{Ge}_{5} \mathrm{Mn}_{2} \mathrm{CrTe}_{8}$ compound. The origin of this pseudo-gap according to Galanakis et al. [28], arise from the hybridization between the nearest neighbors of the $\mathrm{Cr}$-Cr $d$-states and also between the next nearest neighbors $\mathrm{Mn}-\mathrm{Cr} d$-states. Figure $2 \mathrm{a}$ and $\mathrm{b}$ indicate that there is no band gap for the $\mathrm{Ge}_{5} \mathrm{Fe}_{2} \mathrm{CrTe}_{8}$ compound, this demonstrates the metallic character for this compound (metallic bonding).

\subsubsection{Density of states}

The electronic properties of solids were investigated in further detail using the important electronic density of states (DOS) and these quaternary alloys are nearly half-metallic. Figures $3-5$ show the calculated total and partial DOS of the $\mathrm{Ge}_{5} \mathrm{Mn}_{2} \mathrm{CrTe}_{8}, \mathrm{Ge}_{5} \mathrm{Fe}_{2} \mathrm{CrTe}_{8}$, $\mathrm{Ge}_{6} \mathrm{Mn}_{2} \mathrm{Te}_{8}$ and $\mathrm{Ge}_{6} \mathrm{Fe}_{2} \mathrm{Te}_{8}$ alloys using the GGA and the Fermi energy level falls within a region where the magnitude of the spin-down DOS is very small for both alloys. The calculated partial densities of states (PDOS) of the $\mathrm{Cr}, \mathrm{Mn}$ and $\mathrm{Fe}$ atoms in the $\mathrm{Ge}_{5} \mathrm{Mn}_{2} \mathrm{CrTe}_{8}$ and $\mathrm{Ge}_{5} \mathrm{Fe}_{2} \mathrm{CrTe}_{8}$ alloys using the GGA are shown in Figs. 3 and 4 where it is observed that the $\mathrm{Ge}_{5} \mathrm{Mn}_{2} \mathrm{CrTe}_{8}$ compound is half-metallic. In addition, we found that the energy gaps of the minority DOS near the Fermi energy level are mostly dominated by the $\mathrm{Cr} 3 d$ states while the Fe $3 d$ states have a smaller contribution. Figures 3 and
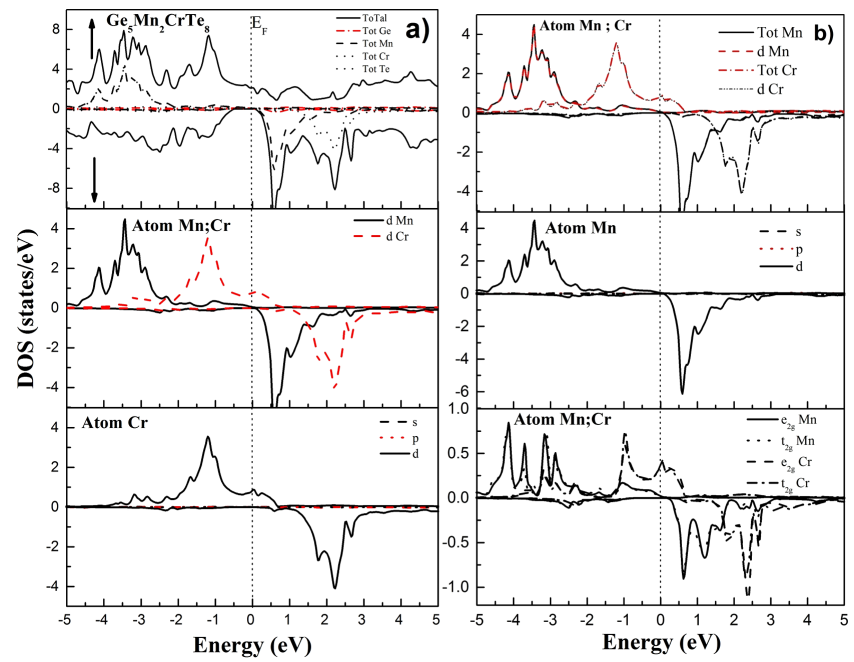

Fig. 3. The calculated total and partial density of states of the $\mathrm{Ge}_{5} \mathrm{Mn}_{2} \mathrm{CrTe}_{8}$ alloy. 


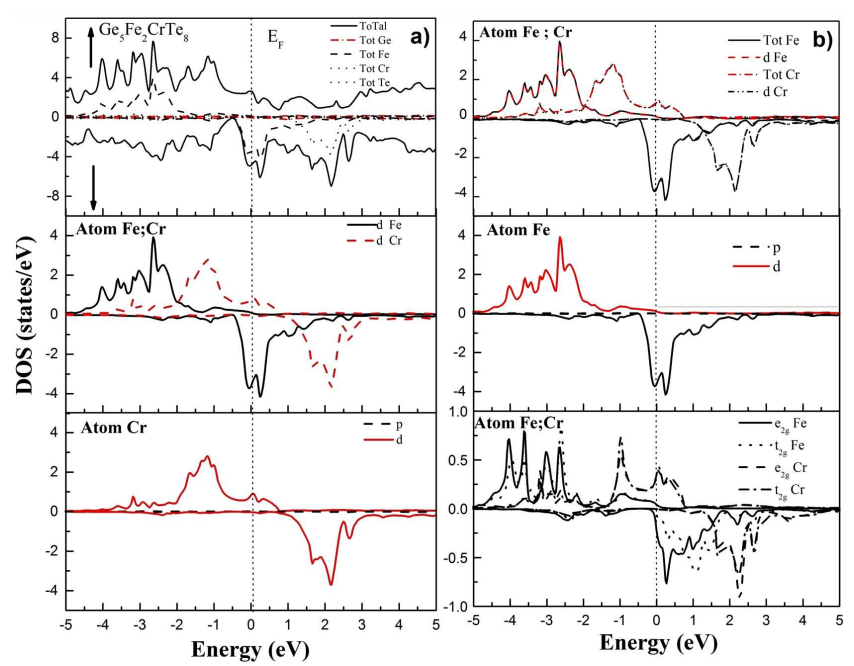

Fig. 4. The calculated total and partial density of states of the $\mathrm{Ge}_{5} \mathrm{Fe}_{2} \mathrm{CrTe}_{8}$ alloy.

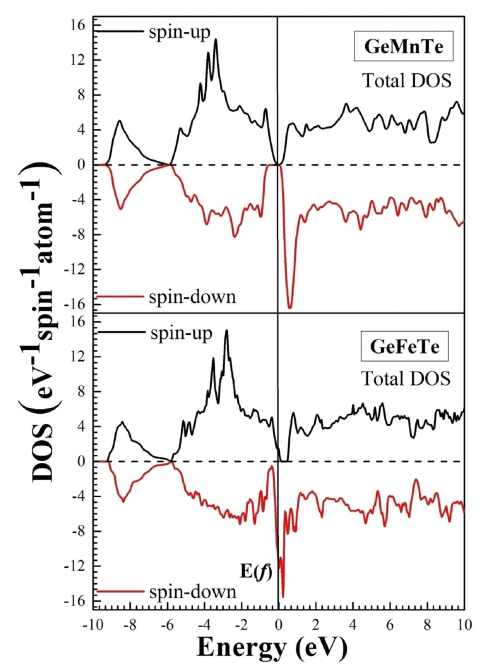

Fig. 5. The calculated total density of states of the $\mathrm{Ge}_{6} \mathrm{Mn}_{2} \mathrm{Te}_{8}$ and $\mathrm{Ge}_{6} \mathrm{Fe}_{2} \mathrm{Te}_{8}$ alloys.

4 exhibit the partial densities of states for the $\mathrm{Cr}$ and Mn $3 d$ states where it is found that the Fermi energy level is mostly dominated by the $\mathrm{Cr} 3 d$ states. As discussed in Ref. [29], the gap is created between the states located exclusively at the $\mathrm{Cr}$ sites.

\subsubsection{Charge densities}

In order to understand the bonding nature in these alloys, the charge distribution of the alloys are examined. Contours maps of the charge density defined on the (111), (110) and (100) planes including a magnetic atom, for the half-metallic $\mathrm{Ge}_{6} \mathrm{Mn}_{2} \mathrm{Te}_{8}$ alloy are calculated to show the bonding characteristics of the Mn atom with the surrounding atoms (see Fig. 6a to c). To explain the nature of the bonding character and the bonding properties of the $\mathrm{Ge}_{6} \mathrm{Mn}_{2} \mathrm{Te}_{8}$ alloy with the RS structure, we have investigated the charge densities in the (111), (110) and (100) planes (see Fig. 6a to c). These figures indicate that there are partial ionic and partial covalent characters in

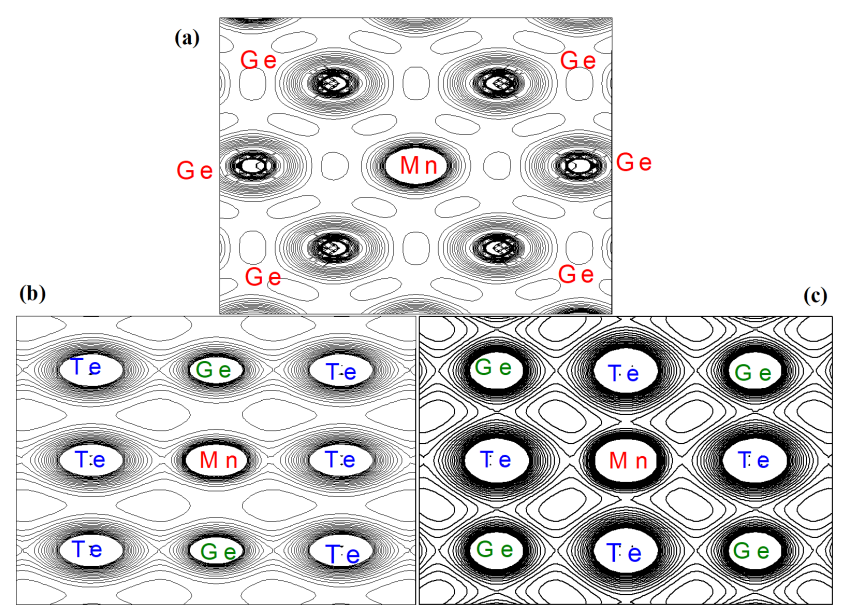

Fig. 6. The charge density distribution of the $\mathrm{Ge}_{6} \mathrm{Mn}_{2} \mathrm{Te}_{8}$ alloy at the equilibrium lattice constant. They are defined on the (a) (111), (b) (110) and the (c) (100) planes, respectively, with the Mn atom being at the centre. The positions of the Ge and Te atoms are also shown in the figures.

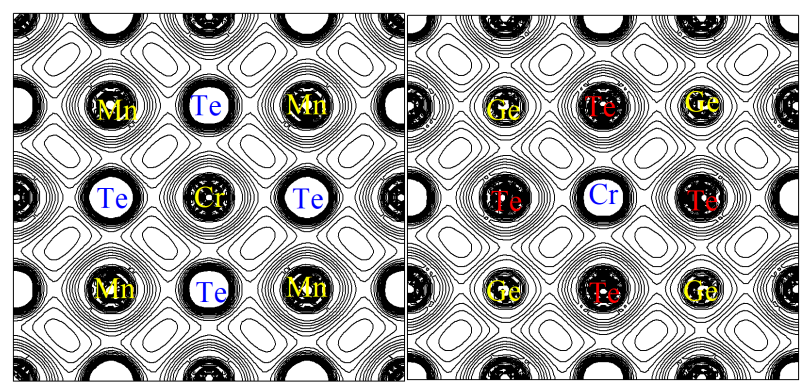

Fig. 7. The charge density distribution of the $\mathrm{Ge}_{5} \mathrm{CrMn}_{2} \mathrm{Te}_{8}$ alloy at the equilibrium lattice constant. They are defined on the (100) plane where the positions of the $\mathrm{Mn}, \mathrm{Ge}, \mathrm{Cr}$ and Te atoms are also shown in the figure.

the alloy where indeed, the interaction between the Ge and Te atoms produce an ionic bonding character due to the $p-d$ hybridization, which is sensitive to temperature (or lattice parameter), whereas the Mn $3 d$ states are relatively localized and are independent of temperature (or lattice parameter). In addition, the displacement of the bonding charges increase as the difference between the electronegativity values of the two atoms increases, which indicates that the $\mathrm{Mn}-\mathrm{Te}$ bond is more ionic as compared to the Ge-Te bond. The charge distributions indicate that the ionic character is predominant in the chemical bonds as shown in Fig. 6a where the Mn atom is surrounded by the Ge atoms, and this feature can be attributed to the hybridization states $p-d$. From Fig. $6 \mathrm{~b}$ and $c$, it is clear that there is a charge transfer between the $\mathrm{Mn}$ and Te atoms and this is due to the difference in the electronegativity which is significant between the Mn and Te atoms (i.e., $\chi_{\mathrm{Te}}-\chi_{\mathrm{Mn}}=0.55$ ), which predicts a partially covalent and partially ionic character. Our results are in agreement with the work by Zhao et al. [10]. 
We can also see the charge density distribution of the $\mathrm{Ge}_{5} \mathrm{CrMn}_{2} \mathrm{Te}_{8}$ alloy at the equilibrium lattice constant where they are defined on the (100) plane. The positions of the Mn, Ge, Cr and Te atoms are shown in Fig. 7. Additionally, when a chemical bond is formed with another element and the difference of the electronegativity is large between the elements, the bond between the atoms tends to be more ionic. Furthermore, our results show that the $\mathrm{Ge}_{6} \mathrm{Mn}_{2} \mathrm{Te}_{8}$ and $\mathrm{Ge}_{5} \mathrm{Mn}_{2} \mathrm{CrTe}_{8}$ alloys are both ionic in nature.

\subsection{Magnetic properties}

\subsubsection{Exchange coupling constants}

From the band structure spectra, we calculate the $p-d$ exchange splitting and the TM $d$-spin exchange splitting energy $\left(\Delta_{x} d\right)$, which is defined as the separation between the corresponding spin-up and spin-down peaks where our results are listed in Table II. The significant parameters determining the magnetic properties of the DMS are the $s-d$ exchange constant $\left(N_{0} \alpha\right)$ and the $p-d$ exchange constant $\left(N_{0 \beta}\right)$, where $N_{0}$ is the concentration of the cations. These parameters describe how the valence and conduction bands contribute to the process of exchange and splitting. From the band structure calculations of the ferromagnetic (FM) $\mathrm{Ge}_{5} \mathrm{Mn}_{2} \mathrm{CrTe}_{8}$ and $\mathrm{Ge}_{5} \mathrm{Fe}_{2} \mathrm{CrTe}_{8}$ alloys, we estimated these exchange coupling constants by assuming the usual Kondo interactions, and $N_{0 \alpha}$ and $N_{0 \beta}$ are defined as [30]:

$$
N_{0 \alpha}=\frac{\Delta E_{c}}{x\langle s\rangle}, N_{0 \beta}=\frac{\Delta E_{v}}{x\langle s\rangle},
$$

where $\Delta E_{v}$ and $\Delta E_{c}$ are the band edge spin splitting of the valence band maxima (VBM) and the conduction band minima (CBM), $x$ is the concentration of the $\mathrm{Mn}$ ( $\mathrm{Cr}, \mathrm{Fe})$ dopants and $\langle s\rangle$ is one-half of the magnetization per TM atom. Our calculated values of $N_{0 \alpha}$ and $N_{0 \beta}$ are given in Table II where we found a negative value of $N_{0 \beta}$ , hence we can deduce that the $p-d$ exchange interaction in the $\mathrm{Ge}_{5} \mathrm{Mn}_{2} \mathrm{CrTe}_{8}$ alloy is antiferromagnetic. Conversely, $N_{0 \beta}$ is found to be -1.16 for the $\mathrm{Ge}_{5} \mathrm{Mn}_{2} \mathrm{CrTe}_{8}$ alloy.

\section{TABLE II}

The calculated spin-exchange splitting energy $\Delta_{x} d(\mathrm{Cr})$ and $\Delta_{x} d(\mathrm{X})$, the calculated conduction and valence band-edge spin-splitting, $\Delta E_{\mathrm{C}}$ and $\Delta E_{v}$ as well as the exchange constants $N_{0 \alpha}$ and $N_{0 \beta}$ (in $\mathrm{eV}$ ) respectively, corresponding to the $\mathrm{Ge}_{5} \mathrm{Mn}_{2} \mathrm{CrTe}_{8}, \mathrm{Ge}_{5} \mathrm{Fe}_{2} \mathrm{CrTe}_{8}$, $\mathrm{Ge}_{6} \mathrm{Mn}_{2} \mathrm{Te}_{8}$ and $\mathrm{Ge}_{6} \mathrm{Fe}_{2} \mathrm{Te}_{8}$ alloys.

\begin{tabular}{c|c|c|c|c|c|c}
\hline \hline Compound & $\Delta_{x} d(\mathrm{Cr})$ & $\Delta_{x} d(\mathrm{X})$ & $\Delta \mathbf{E}_{\mathbf{c}}$ & $\Delta E_{v}$ & $N_{0 \alpha}$ & $N_{0 \beta}$ \\
\hline $\mathrm{Ge}_{5} \mathrm{Mn}_{2} \mathrm{CrTe}_{8}$ & 3.24 & 4.09 & 0.15 & -0.45 & 0.24 & -1.16 \\
$\mathrm{Ge}_{5} \mathrm{Fe}_{2} \mathrm{CrTe}_{8}$ & 3.06 & 2.65 & 0 & 0.133 & 0 & 0.35 \\
$\mathrm{Ge}_{6} \mathrm{Mn}_{2} \mathrm{Te}_{8}$ & - & 4.12 & -0.15 & -0.39 & -0.24 & -0.62 \\
$\mathrm{Ge}_{6} \mathrm{Fe}_{2} \mathrm{Te}_{8}$ & - & 4.03 & -0.35 & -0.33 & -0.93 & -0.88
\end{tabular}

On the other hand, we found that the TM-3d spin exchange splitting energy $\left(\Delta_{x} d\right)$, which is defined as the separation between the corresponding spin up and spin down peaks, are $4.12 \mathrm{eV}$ and $4.03 \mathrm{eV}$ for the $\mathrm{Ge}_{6} \mathrm{Mn}_{2} \mathrm{Te}_{8}$ and $\mathrm{Ge}_{6} \mathrm{Fe}_{2} \mathrm{Te}_{8}$ alloys, respectively. In addition, the $\left(\Delta_{x} d\right)$ values are $4.09,3.24$, and $2.65,3.06 \mathrm{eV}$ for the $\mathrm{Ge}_{5} \mathrm{Mn}_{2} \mathrm{CrTe}_{8}$ and $\mathrm{Ge}_{5} \mathrm{Fe}_{2} \mathrm{CrTe}_{8}$ alloys, respectively.
Another important exchange splitting parameter, which illustrates the nature of the attraction in these alloys is the $p-d$ exchange splitting parameter at the maxima of the valence bands for the spin up and spin down cases given by

$$
\Delta_{x}(p d)=E_{v}(\downarrow)-E_{v}(\uparrow),
$$

where $E_{v}(\downarrow)$ and $E_{v}(\uparrow)$ are the valence band maxima of the minority spin and majority spin, respectively. The obtained negative $\Delta_{x}(p d)$ values are -0.39 , -0.33 , and $-0.45 \mathrm{eV}$ for the $\mathrm{Ge}_{6} \mathrm{Mn}_{2} \mathrm{Te}_{8}, \mathrm{Ge}_{6} \mathrm{Fe}_{2} \mathrm{Te}_{8}$ and $\mathrm{Ge}_{5} \mathrm{Mn}_{2} \mathrm{CrTe}_{8}$ alloys, respectively, which indicate that the effective potential for the minority spin is more attractive as compared to the majority spin, which characterizes the spin polarized systems [31].

\subsubsection{Magnetic moments}

In Table III, we summarized the calculated total and local magnetic moments per atom within the muffin-tin spheres for the $\mathrm{Ge}_{5} \mathrm{Mn}_{2} \mathrm{CrTe}_{8}$ and $\mathrm{Ge}_{5} \mathrm{Fe}_{2} \mathrm{CrTe}_{8}$ alloys where the results show that the total magnetic moments come from the $\mathrm{Mn}, \mathrm{Fe}$ and $\mathrm{Cr}$ ions. The $\mathrm{Cr}$ atoms are ferromagnetically coupled to the Mn spin moments and they possess a spin moment that varies in a manner as observed: when we doped $\mathrm{Cr}$ in the $\mathrm{Ge}_{6} \mathrm{Mn}_{2} \mathrm{Te}_{8}$ and $\mathrm{Ge}_{6} \mathrm{Fe}_{2} \mathrm{Te}_{8}$ systems, we see that the total magnetic moments increase from $5.003 \mu_{\mathrm{B}}$ to $9.001 \mu_{\mathrm{B}}$ for Cr doping in the $\mathrm{Ge}_{6} \mathrm{Mn}_{2} \mathrm{Te}_{8}$ alloy and from $3.900 \mu_{\mathrm{B}}$ to $7.673 \mu_{\mathrm{B}}$ for $\mathrm{Cr}$ doping in the $\mathrm{Ge}_{6} \mathrm{Fe}_{2} \mathrm{Te}_{8}$ alloy. According to Table III, the $p-d$ hybridization near the Fermi level decreases the atomic magnetic moment of the $\mathrm{Mn}$ and $\mathrm{Fe}$ atoms from their atomic charge values and create small amount of local magnetic moments on the nonmagnetic Ge and Te sites.

TABLE III

The calculated total and local magnetic moments (in $\mu_{\mathrm{B}}$ ) within the muffin tin spheres and in the interstitial sites of the $\mathrm{Ge}_{5} \mathrm{Mn}_{2} \mathrm{CrTe}_{8}$ and $\mathrm{Ge}_{5} \mathrm{Fe}_{2} \mathrm{CrTe}_{8}$ alloys.

\begin{tabular}{c|c|c|c|c|c|c}
\hline \hline Compound & Ge & Mn & Cr & Te & Interstitial & Total \\
\hline $\mathrm{Ge}_{6} \mathrm{Mn}_{2} \mathrm{Te}_{8}$ & 0.014 & 4.057 & - & 0.028 & 0.815 & 5.003 \\
$\mathrm{Ge}_{5} \mathrm{Mn}_{2} \mathrm{CrTe}_{8}$ & 0.037 & 4.050 & 3.399 & 0.014 & 1.523 & 9.001 \\
$\mathrm{Ge}_{6} \mathrm{Fe}_{2} \mathrm{Te}_{8}$ & 0.030 & 3.137 & - & 0.052 & 0.552 & 3.900 \\
$\mathrm{Ge}_{5} \mathrm{Fe}_{2} \mathrm{CrTe}_{8}$ & 0.039 & 3.079 & 3.322 & 0.004 & 1.186 & 7.673
\end{tabular}

\subsection{Thermal properties}

The thermal properties of a material determine the suitable conditions for initiating and maintaining the quality of the device fabrication. In order to investigate the thermal properties of the $\mathrm{Ge}_{5} \mathrm{Mn}_{2} \mathrm{CrTe}_{8}$ and $\mathrm{Ge}_{5} \mathrm{Fe}_{2} \mathrm{CrTe}_{8}$ alloys under high pressure and high temperature, we have used the Gibbs program [27] to determine all the thermodynamic parameters as a function of temperature and pressure, which were used to determine other macroscopic properties $[27,32]$ of the alloys. This program is based on the quasi-harmonic Debye model, in which the non-equilibrium Gibbs function, $G^{*}(V ; P ; T)$ takes the form of

$$
G^{*}(V ; P ; T)=E(V)+P V+A_{v i b}(\theta(V) ; T),
$$
where $E(V)$ is the total energy per unit cell, $P V$ corresponds to the constant hydrostatic pressure condition, 
$A_{v i b}$ is the vibrational Helmholtz free energy, and $\theta$ is the Debye temperature which can be written as [33, 34]:

$$
\begin{aligned}
& A_{v i b}(\theta(V) ; T)= \\
& \quad n K T\left(\frac{9 \theta}{8 T}+3 \ln \left(1-\mathrm{e}^{-\frac{\theta}{T}}\right)-D\left(\frac{\theta}{T}\right)\right) .
\end{aligned}
$$

It is related to an average sound velocity since in the Debye theory the vibrations of the solid are considered as elastic waves. Assuming an isotropic solid with the Poisson ratio [35], the parameter $\theta$ is expressed as [36]:

$$
\theta=h / 2 \pi k \sqrt[3]{6 \pi^{2} V^{1 / 2} n} /^{3} f(\nu) \sqrt{B s / M} .
$$

Since $B_{s}$ measures the compressibility of the crystal for the fixed quantum state populations, it can be approximated by the static compressibility where its general dependence on $x$ can be further simplified as [27]:

$$
\begin{aligned}
& B_{s}=V\left(\mathrm{~d}^{2} E(V) / \mathrm{d} V^{2}\right), \\
& \left(\sigma G^{*}(V ; P ; T) / \sigma V\right)_{P ; T}=0 .
\end{aligned}
$$

By solving Eq. (7), the isothermal bulk modulus $B T$ and the other thermal properties of the alloys such as the heat capacity at constant volume $C_{V}$, the heat capacity at constant pressure $C_{p}$, and the thermal expansion $\alpha$ are respectively taken as [27]:

$$
\begin{aligned}
& C_{V}=3 n k\left(4 D\left(\frac{\theta}{T}\right)-3 \frac{\theta}{T}\left(\mathrm{e}^{\frac{\theta}{T}}-1\right)\right), \\
& C_{p}=C_{V}(1+\alpha \gamma T), \\
& \alpha=\gamma C_{V} / B_{T} V,
\end{aligned}
$$

where $\gamma$ represents the Grüneisen parameter, which is expressed as

$$
\gamma=-(\mathrm{d} \ln \theta(V) / \mathrm{d} \ln V) \text {. }
$$

Based on the quasi-harmonic Debye model, we have investigated the thermal properties of the $\mathrm{Ge}_{5} \mathrm{Mn}_{2} \mathrm{CrTe}_{8}$ and $\mathrm{Ge}_{5} \mathrm{Fe}_{2} \mathrm{CrTe}_{8}$ alloys over a range of pressures from $0 \mathrm{GPa}$ to $18 \mathrm{GPa}$. The temperature and pressure dependences of the bulk modulus $B$ for the $\mathrm{Ge}_{5} \mathrm{Mn}_{2} \mathrm{CrTe}_{8}$ and $\mathrm{Ge}_{5} \mathrm{Fe}_{2} \mathrm{CrTe}_{8}$ alloys are plotted in Fig. 8 where it can be seen from Fig. 8a that $B$ decreases with increase of $T$ at a given pressure, while $B$ increases with increase of $P$ at a given temperature. Furthermore, it also shows that the effect of increase of pressure on the $\mathrm{Ge}_{5} \mathrm{Mn}_{2} \mathrm{CrTe}_{8}$ and $\mathrm{Ge}_{5} \mathrm{Fe}_{2} \mathrm{CrTe}_{8}$ alloys is the same as that of decrease of the temperature. However, Fig. $8 \mathrm{~b}$ also shows that the effect of the pressure is more important as compared to the effect of the temperature on the bulk modulus where Fig. $8 \mathrm{~b}$ shows that at zero pressure, the bulk modulus $B$ is nearly constant at low temperature, whereas the value of $B$ decreases dramatically with the increase of temperature for the $\mathrm{Ge}_{5} \mathrm{Fe}_{2} \mathrm{CrTe}_{8}$ alloy. However, for the $\mathrm{Ge}_{5} \mathrm{Mn}_{2} \mathrm{CrTe}_{8}$ alloy, we find that at zero pressure, the bulk modulus $B$ is nearly constant at low temperature, whereas the value of $B$ increases dramatically with the increase of temperature.

The primitive cell volume $\left(V / V_{0}\right)$ of the $\mathrm{Ge}_{5} \mathrm{Mn}_{2} \mathrm{CrTe}_{8}$ and $\mathrm{Ge}_{5} \mathrm{Fe}_{2} \mathrm{CrTe}_{8}$ alloys as a function of pressure $P$ at $T=0,200,400,600,800$, and $1000 \mathrm{~K}$ are shown in Fig. 9. Correspondingly, in Fig. 9 when $T<200 \mathrm{~K}$, the primitive cell volume of the $\mathrm{Ge}_{5} \mathrm{Mn}_{2} \mathrm{CrTe}_{8}$ and $\mathrm{Ge}_{5} \mathrm{Fe}_{2} \mathrm{CrTe}_{8}$ alloys

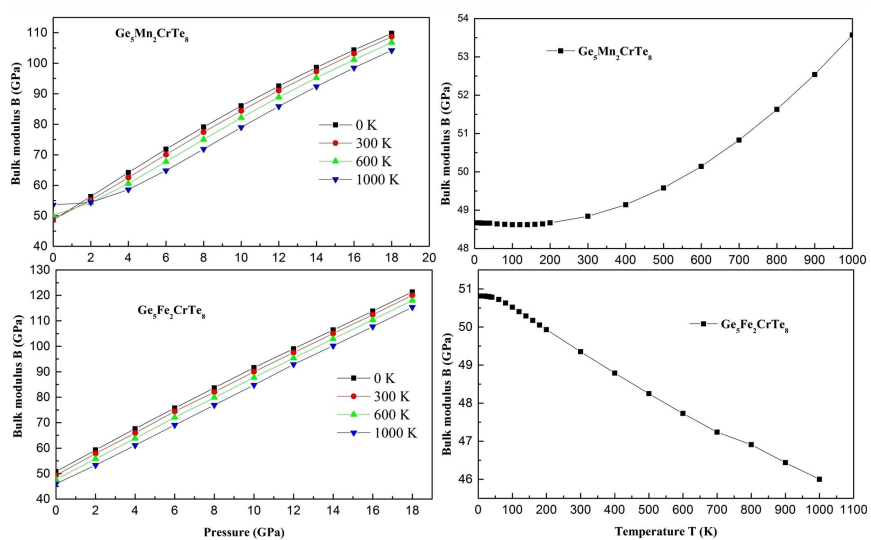

Fig. 8. The pressure and temperature dependence of the bulk modulus $B$ of the $\mathrm{Ge}_{5} \mathrm{Fe}_{2} \mathrm{CrTe}_{8}$ alloy.

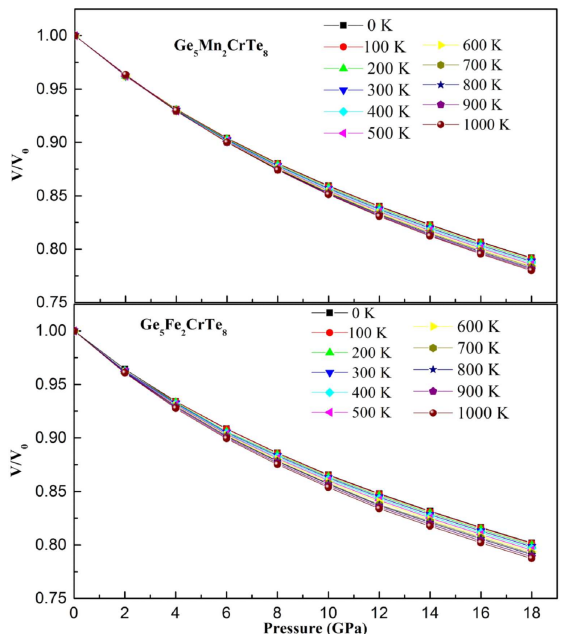

Fig. 9. The primitive cell volume $\left(V / V_{0}\right)$ of the $\mathrm{Ge}_{5} \mathrm{Mn}_{2} \mathrm{CrTe}_{8}$ and $\mathrm{Ge}_{5} \mathrm{Fe}_{2} \mathrm{CrTe}_{8}$ alloys as a function of pressure $P$ at $T=0,200,400,600,800$, and $1000 \mathrm{~K}$.

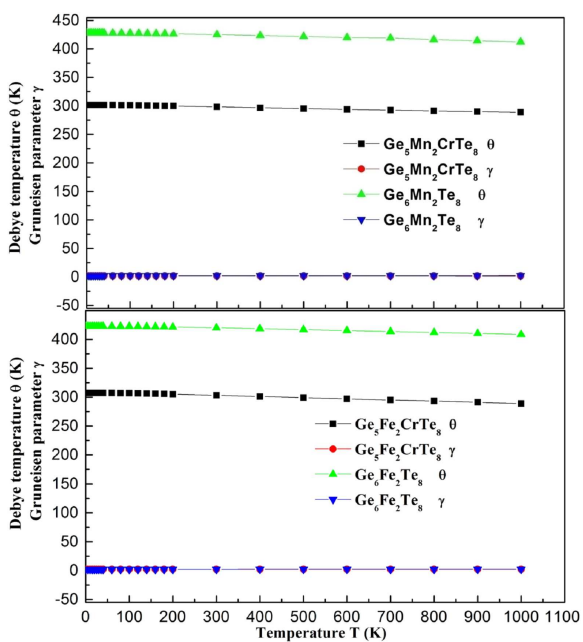

Fig. 10. The variation of the Debye temperature $\theta_{\mathrm{D}}(\mathrm{K})$ and Grüneisen constant $\gamma$ versus temperature $T$ (K) at zero pressure for the $\mathrm{Ge}_{6} \mathrm{Mn}_{2} \mathrm{Te}_{8}, \mathrm{Ge}_{5} \mathrm{Mn}_{2} \mathrm{CrTe}_{8}$, $\mathrm{Ge}_{6} \mathrm{Fe}_{2} \mathrm{Te}_{8}$ and $\mathrm{Ge}_{5} \mathrm{Fe}_{2} \mathrm{CrTe}_{8}$ alloys. 
exhibits little changes. However, when the temperature is greater than $200 \mathrm{~K}$ the cell volume change significantly as $T$ increases. This is because of the rapid variation in the cell volume which causes $B$ to decrease rapidly. From Fig. 9, it is observed that the effect of temperature on the ratio $V / V_{0}$ in the alloys is not as significant as compared to the effect of pressure in our calculated pressure and temperature ranges.

The Debye temperature $\left(\theta_{\mathrm{D}}\right)$ is an important parameter for indicating the characteristics of the thermal properties of solids. For temperatures above $\theta_{\mathrm{D}}$, the crystal behaves classically, due to the thermal vibrations becoming more important than the quantum effects. Figure 10 shows the variation of $\theta_{\mathrm{D}}$ with temperature where it can be observed that $\theta_{\mathrm{D}}$ is nearly constant from $0 \mathrm{~K}$ to $100 \mathrm{~K}$ and for $T>100 \mathrm{~K}, \theta_{\mathrm{D}}$ decreases linearly with increase of temperature. In addition, the calculated Debye temperatures of the $\mathrm{Ge}_{6} \mathrm{Mn}_{2} \mathrm{Te}_{8}, \mathrm{Ge}_{5} \mathrm{Mn}_{2} \mathrm{CrTe}_{8}, \mathrm{Ge}_{6} \mathrm{Fe}_{2} \mathrm{Te}_{8}$, and $\mathrm{Ge}_{5} \mathrm{Fe}_{2} \mathrm{CrTe}_{8}$ alloys at room temperature and zero pressure are $425.21,298.25,420.20$, and $303.19 \mathrm{~K}$, respectively.

The Grüneisen constant $\gamma$ describes the anharmonic effects in the vibrating lattice and indicates the volume dependence of the Debye temperature $\theta$. Additionally, $\gamma$ can also correctly predict the anharmonic properties of a material where Fig. 10 shows the variation of $\gamma$ with temperature. It can be observed that $\gamma$ is nearly constant from $0 \mathrm{~K}$ to $100 \mathrm{~K}$ and for $T>100 \mathrm{~K}, \gamma$ increases linearly with increase of temperature. Furthermore, the calculated Grüneisen constant of the $\mathrm{Ge}_{6} \mathrm{Mn}_{2} \mathrm{Te}_{8}$, $\mathrm{Ge}_{5} \mathrm{Mn}_{2} \mathrm{CrTe}_{8}, \mathrm{Ge}_{6} \mathrm{Fe}_{2} \mathrm{Te}_{8}$ and $\mathrm{Ge}_{5} \mathrm{Fe}_{2} \mathrm{CrTe}_{8}$ alloys at room temperature and zero pressure are 2.229, 1.794, 2.1, and 2.009 , respectively.

The heat capacities of a material are very important parameters which not only provide crucial insights into the vibrational properties of the material but the values of these parameters are also essential for many applications. The variation of the specific heat at constant volume $C_{V}$ versus temperature $T$ at zero pressure of the alloys is shown in Fig. 11 where it can be observed that $C_{V}$ increases with $T^{3}$ [37] at lower temperature, whereas with the increase of temperature, $C_{V}$ follows the Debye model and approaches the Dulong-Petit limit [38]. This indicates that the thermal energy at high temperature excites all the phonon modes, which is common to all solids at high temperature. At room temperature and zero pressure, the $C_{V}$ values of the $\mathrm{Ge}_{6} \mathrm{Mn}_{2} \mathrm{Te}_{8}$, $\mathrm{Ge}_{5} \mathrm{Mn}_{2} \mathrm{CrTe}_{8}, \mathrm{Ge}_{6} \mathrm{Fe}_{2} \mathrm{Te}_{8}$ and $\mathrm{Ge}_{5} \mathrm{Fe}_{2} \mathrm{CrTe}_{8}$ alloys are 90.42, 95.01, 90.63, and 94.86 $\mathrm{J} \mathrm{mol}^{-1} \mathrm{~K}^{-1}$, respectively.

The relationship between entropy $S$ and temperature $T$ is displayed in Fig. 12 where it is observed that at zero pressure, the entropy $S$ generally increases exponentially with the temperature $T$. In addition, it can also be observed that the effects of temperature on the entropy are more important at high temperature as compared to at low temperature. At $300 \mathrm{~K}$ and zero pressure, the obtained value of entropy is $103.067,136.04,104.14$, and $134.48 \mathrm{~J} \mathrm{~mol}^{-1} \mathrm{~K}^{-1}$ for the $\mathrm{Ge}_{6} \mathrm{Mn}_{2} \mathrm{Te}_{8}, \mathrm{Ge}_{5} \mathrm{Mn}_{2} \mathrm{CrTe}_{8}$,

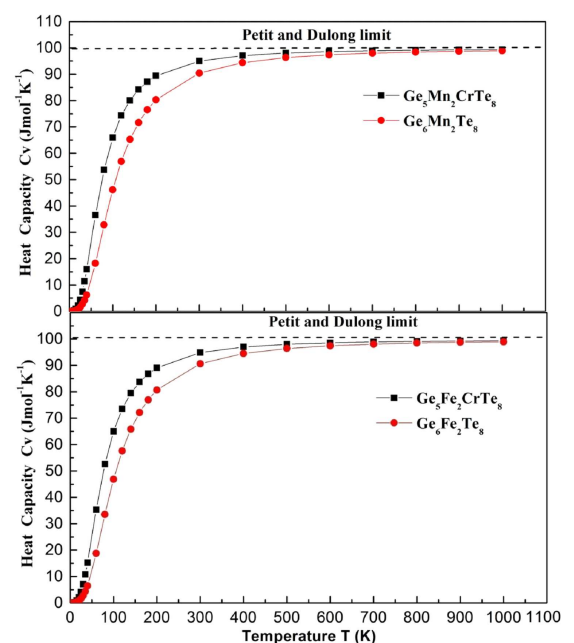

Fig. 11. The variation of the heat capacity, $C_{V}$ $\left(\mathrm{J} \mathrm{mol}^{-1} \mathrm{~K}^{-1}\right)$ versus temperature $T(\mathrm{~K})$ at zero pressure of the $\mathrm{Ge}_{6} \mathrm{Mn}_{2} \mathrm{Te}_{8}, \mathrm{Ge}_{5} \mathrm{Mn}_{2} \mathrm{CrTe}_{8}, \mathrm{Ge}_{6} \mathrm{Fe}_{2} \mathrm{Te}_{8}$ and $\mathrm{Ge}_{5} \mathrm{Fe}_{2} \mathrm{CrTe}_{8}$ alloys.

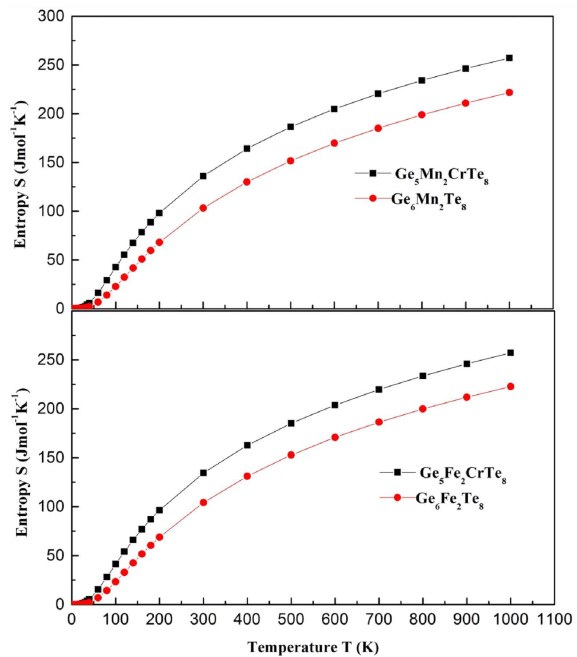

Fig. 12. The variation of the entropy $S\left(\mathrm{~J} \mathrm{~mol}^{-1} \mathrm{~K}^{-1}\right)$ versus temperature $T(\mathrm{~K})$ at zero pressure of the $\mathrm{Ge}_{6} \mathrm{Mn}_{2} \mathrm{Te}_{8}, \mathrm{Ge}_{5} \mathrm{Mn}_{2} \mathrm{CrTe}_{8}, \mathrm{Ge}_{6} \mathrm{Fe}_{2} \mathrm{Te}_{8}$ and $\mathrm{Ge}_{5} \mathrm{Fe}_{2} \mathrm{CrTe}_{8}$ alloys.

$\mathrm{Ge}_{6} \mathrm{Fe}_{2} \mathrm{Te}_{8}$, and $\mathrm{Ge}_{5} \mathrm{Fe}_{2} \mathrm{CrTe}_{8}$ alloys, respectively.

Within the quasi-harmonic approximation, the anharmonicity is restricted to the thermal expansion and Fig. 13a and b shows the volume thermal expansion coefficient $(\alpha)$ dependence on temperature and pressure. It can be seen in Fig. 15a that at a given temperature, the volume thermal expansion coefficient $\alpha$ rapidly increases, especially at zero pressure and below the temperature at $300 \mathrm{~K}$. Subsequently, the thermal expansion coefficient gradually tends to increase linearly at the higher temperature region which is due to the electronic contribution. However, it is also observed from Fig. 15b that as the pressure increases, the volume thermal expansion coefficient decreases significantly at $300 \mathrm{~K}$. Therefore, these results indicate that the anhar- 


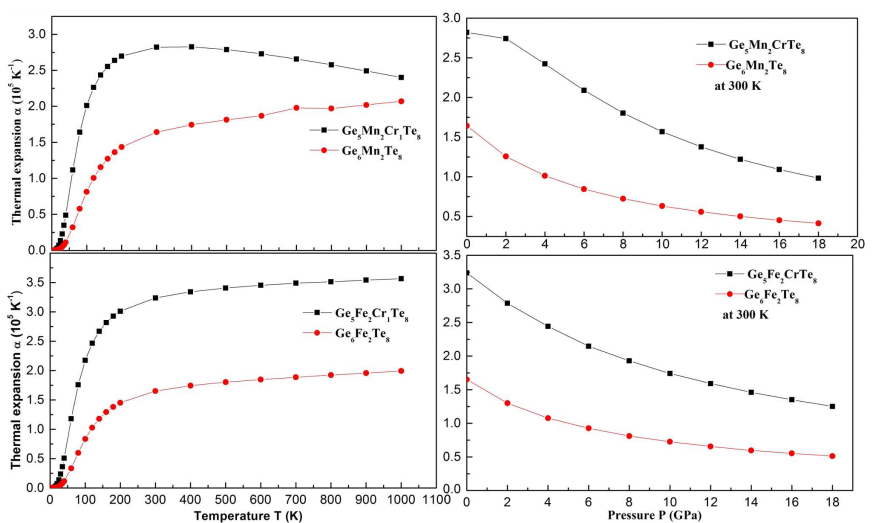

Fig. 13. The variation of the (a) thermal expansion $\alpha$ versus temperature at zero pressure and also (b) the variation of the thermal expansion $\alpha$ versus pressure (GPa) at $300 \mathrm{~K}$ of the $\mathrm{Ge}_{6} \mathrm{Mn}_{2} \mathrm{Te}_{8}, \mathrm{Ge}_{5} \mathrm{Mn}_{2} \mathrm{CrTe}_{8}$, $\mathrm{Ge}_{6} \mathrm{Fe}_{2} \mathrm{Te}_{8}$ and $\mathrm{Ge}_{5} \mathrm{Fe}_{2} \mathrm{CrTe}_{8}$ alloys.

monic effects are important at low temperatures and high pressures for the $\mathrm{Ge}_{6} \mathrm{Mn}_{2} \mathrm{Te}_{8}, \mathrm{Ge}_{5} \mathrm{Mn}_{2} \mathrm{CrTe}_{8}, \mathrm{Ge}_{6} \mathrm{Fe}_{2} \mathrm{Te}_{8}$ and $\mathrm{Ge}_{5} \mathrm{Fe}_{2} \mathrm{CrTe}_{8}$ alloys. Meanwhile, at $300 \mathrm{~K}$ and zero pressure, the volume thermal expansion coefficient, $\alpha$ of the $\mathrm{Ge}_{6} \mathrm{Mn}_{2} \mathrm{Te}_{8}, \mathrm{Ge}_{5} \mathrm{Mn}_{2} \mathrm{CrTe}_{8}, \mathrm{Ge}_{6} \mathrm{Fe}_{2} \mathrm{Te}_{8}$ and $\mathrm{Ge}_{5} \mathrm{Fe}_{2} \mathrm{CrTe}_{8}$ alloys are $1.64 \times 10^{5}, 2.82 \times 10^{5}, 1.65 \times 10^{5}$, and $3.24 \times 10^{5} \mathrm{~K}^{-1}$, respectively, and $\alpha$ rapidly decreases with increasing pressure from $2 \mathrm{GPa}$ to $18 \mathrm{GPa}$. This might be an indication that the quasi-harmonic Debye model is a very reasonable alternative model to account for the thermal effects in the alloys with relatively inexpensive computational time.

\section{Summary and conclusions}

In conclusion, we have performed first principle calculations based on the FP-LAPW + LO method within the GGA to evaluate the structural, electronic, magnetic and thermal properties of the quaternary $\mathrm{Ge}_{5} \mathrm{Mn}_{2} \mathrm{CrTe}_{8}$ and $\mathrm{Ge}_{5} \mathrm{Fe}_{2} \mathrm{CrTe}_{8}$ alloys. From the calculations, it was observed that the optimized lattice parameters agree well with the available experimental data for the investigated alloys and the results indicate that both quaternary alloys are half-metallic. Furthermore, the results of the exchange splittings, $\Delta_{x}(p d)$ parameters due to the Mn $3 d$ states show that the effective potential for the minority spin is more attractive as compared to the majority spin. A negative value of $N_{0 \beta}$ can be used to deduce that the $p-d$ exchange interaction in the $\mathrm{Ge}_{5} \mathrm{Mn}_{2} \mathrm{CrTe}_{8}$ alloy is antiferromagnetic and the total magnetic moments increase when we doped $\mathrm{Cr}$ in the $\mathrm{Ge}_{6} \mathrm{Mn}_{2} \mathrm{Te}_{8}$ and $\mathrm{Ge}_{6} \mathrm{Fe}_{2} \mathrm{Te}_{8}$ systems. Our results of the calculations of the charge density show that the bonding nature of the $\mathrm{Ge}_{6} \mathrm{Mn}_{2} \mathrm{Te}_{8}$ and $\mathrm{Ge}_{5} \mathrm{Mn}_{2} \mathrm{CrTe}_{8}$ alloys are both ionic. Using the quasi-harmonic Debye model, the characteristics of the bulk modulus $B$, the primitive cell volume $V / V_{0}$, the Debye temperature $\theta_{\mathrm{D}}$, the Grüneisen parameter $\gamma$, the heat capacity $C_{V}$, the entropy $S$ and the thermal expansion coefficient $\alpha$ versus pressure and temperature of the $\mathrm{Ge}_{6} \mathrm{Mn}_{2} \mathrm{Te}_{8}, \mathrm{Ge}_{5} \mathrm{Mn}_{2} \mathrm{CrTe}_{8}, \mathrm{Ge}_{6} \mathrm{Fe}_{2} \mathrm{Te}_{8}$ and $\mathrm{Ge}_{5} \mathrm{Fe}_{2} \mathrm{CrTe}_{8}$ alloys have been obtained successfully and were also investigated in detail, as these parameters are essential for thin film growth. Presently, since there are no available experimental data for these quantities, we feel that the $a b$ initio theoretical method is the only reasonable tool or approach for obtaining important information of the alloys and our calculated results may provide the reference data for future experimental work.

\section{References}

[1] W. Knoff, A. Łusakowski, A. Wołoś, T. Story, Acta Phys. Pol. A 127, 404 (2015).

[2] A. Podgórni, L. Kilanski, W. Dobrowolski, M. Górska, V. Domukhovski, B. Brodowska, A. Reszka, B.J. Kowalski, V.E. Slynko, E.I. Slynko, Acta Phys. Pol. A 126, 1180 (2014).

[3] V.N. Antonov, A.P. Shpak, L.V. Bekenov, L.P. Germash, A.N. Yaresko, Condens. Matter Phys. 13 , 23702 (2010).

[4] M.D. Gustavo, S.H. Wei, X.G. Gong, A.J.R. da Silva, A. Fazzio, Solid State Commun. 138, 353 (2006).

[5] W.Q. Chen, S.T. Lim, C.H. Sim, J.F. Bi, K.L. Teo, T. Liew, T.C. Chong, J. Appl. Phys. 104, 063912 (2008).

[6] Y. Fukuma, T. Murakami, H. Asada, T. Koyanagi, Physica E 10, 273 (2001).

[7] Y. Fukuma, H. Asada, J. Miyashita, N. Nishimura, T. Koyanagi, J. Appl. Phys. 93, 7667 (2003).

[8] Y. Fukuma, H. Asada, S. Miyawaki, T. Koyanagi, S. Senba, K. Goto, H. Sato, Appl. Phys. Lett. 93, 252502 (2008).

[9] Y. Fukuma, H. Asada, T. Taya, T. Irisa, T. Koyanagi, Appl. Phys. Lett. 89, 152506 (2006).

[10] Y.H. Zhao, W.H. Xie, L.F. Zhu, B.G. Liu, J. Phys. Condens. Matter 18, 10259 (2006).

[11] A. Ciucivara, B.R. Sahu, L. Kleinman, Phys. Rev. B 75, 241201(R) (2007).

[12] K.M. Wong, Results Phys. 7, 1308 (2017).

[13] K.M. Wong, Jpn. J. Appl. Phys. 48, 085002 (2009).

[14] K.M. Wong, W.K. Chim, J.Q. Huang, L. Zhu, J. Appl. Phys. 103, 054505 (2008).

[15] Y. Fukuma, H. Asada, N. Moritake, T. Irisa, T. Koyanagi, Appl. Phys. Lett. 91, 092501 (2007).

[16] L. Kilanski, R. Szymczak, W. Dobrowolski, K. Szałowski, V.E. Slynko, E.I. Slynko, Phys. Rev. B 82, 094427 (2010).

[17] N. Baki, S. Mécabih, H. Khachai, B. Abbar, J. Magn. Magn. Mater. 345, 222 (2013).

[18] K. Sato, L. Bergqvist, J. Kudrnovský, P.H. Dederichs, O. Eriksson, I. Turek, B. Sanyal, G. Bouzerar, H. Katayama-Yoshida, V.A. Dinh, T. Fukushima, H. Kizaki, R. Zeller, Rev. Mod. Phys. 82, 1633 (2010).

[19] L. Bergqvist, O. Eriksson, J. Kudrnovský, V. Drchal, P. Korzhavyi, I. Turek, Phys. Rev. Lett. 93, 137202 (2004).

[20] Y. Liu, S.K. Bose, J. Kudrnovský, J. Appl. Phys. 112, $053902(2012)$ 
[21] P. Blaha, K. Schwarz, G.K.H. Madsen, D. Kvasnicka, J. Luitz, WIEN2K, An Augmented Plane Wave Plus Local Orbitals Program for Calculating Crystal Properties, Vienna University of Technology, Austria 2014.

[22] K.M. Wong, M. Irfan, A. Mahmood, G. Murtaza, J. Electron. Mater. s11664-017-5805-1 (2017).

[23] K.M. Wong, S.M. Alay-e-Abbas, Y. Fang, A. Shaukat, Y. Lei, J. Appl. Phys. 114, 034901 (2013).

[24] J.P. Perdew, A. Ruzsinszky, G. Csonka, O.A. Vydrov, G.E. Scuseria, L.A. Constantin, X. Zhou, K. Burke, Phys. Rev. Lett. 100, 136406 (2008).

[25] F.D. Murnaghan, Proc. Natl. Acad. Sci. USA 30, 244 (1944).

[26] H.J. Monkhorst, J.D. Pack, Phys. Rev. B 13, 5188 (1976).

[27] M.A. Blanco, E. Francisco, V. Luańa, Comput. Phys. Commun. 158, 57 (2004).

[28] I. Galanakis, P.H. Dederichs, N. Papanikolaou, Phys. Rev. B 66, 174429 (2002).

[29] S. Ishida, S. Kashiwagi, S. Fujii, S. Asano, Physica $B$ 210, 140 (1995).
[30] V.L. Moruzzi, T.F. Janak, A.R. Williams, Calculated Electronic Propertied of Metals, Pergamon, New York 1978.

[31] J.A. Gaj, R. Planel, G. Fishman, Solid State Commun. 29, 435 (1979).

[32] E. Francisco, M.A. Blanco, G. Sanjurjo, Phys. Rev. B 63, 094107 (2001).

[33] M.A. Blanco, A.M. Pendás, E. Francisco, J.M. Recio, R. Franco, Comput. Theor. Chem. 368, 245 (1996).

[34] M. Florez, J.M. Recio, E. Francisco, M.A. Blanco, A.M. Pendás, Phys. Rev. B 66, 144112 (2002).

[35] J.P. Poirier, Introduction to the Physics of the Earth's Interior, 2nd ed., Cambridge University Press, England 2000.

[36] M.A. Blanco, PhD Thesis, Universidad de Oviedo, Spain 1997.

[37] P. Debye, Ann. Phys. 39, 789 (1912).

[38] A.T. Petit, P.L. Dulong, Ann. Chim. Phys. 10, 395 (1819). 